\title{
EDITORIAL
}

\section{New pedagogies for e-learning?}

\section{Martin Oliver}

\section{Institute of Education, UK}

The question of how technology affects pedagogy is not new, but has started to become a focus for attention again. The UK's Joint Information Systems Committee, for example, has spent the past two years supporting a programme of research into this topic, both from the perspective of the teacher (understanding designing for learning) and more recently from the perspective of the learner (understanding the learner's experience).

What is different, this time around? The promise of technology to revolutionise teaching has a long-established history that seems to have failed to materialise. In the mid-1950s, Skinner proposed the idea of the teaching machine; even earlier, the US military had made a serious commitment into what would become educational technology by looking at the potential of video for training. We can chart a long series of developments since-the microcomputer, computer-based training, multimedia, the web. And yet we are still trying to understand this relationship between technology and learning.

Meanwhile, the media debate has provided background noise-McLuhan's claim that the medium is the message and the counter-arguments that the medium has no effect on message content confusing matters still further. Which is more naïve: to look for an effect of technology on learning, or to assume that there will be none?

I was recently involved in a small European project that set out to explore the relationship between technology use and the roles that teachers undertook. As part of this, a colleague (Sara Price, Institute of Education) and I analysed some interviews undertaken with lecturers who were starting to use virtual learning environments for the first time. Perhaps, in retrospect, it should have been obvious-but our analysis suggested that, for these people, teaching with a new medium was the same and completely different simultaneously. It all depended upon how they framed teaching. When they talked about it strategically, nothing changed: they still had to conceive the curriculum, monitor students' progress, provide feedback, and so on. At an intermediate level, the actions they undertook were slightly different: they would check contributions to a bulletin board, for example, instead of trying to spot who was sitting quietly on the edges of the seminar; different tools were used, but the practices were recognisable. At a fine-grained level of analysis, none of their original practices 


\section{Editorial}

remained. Button clicks and typing replaced conversation; automated records replaced sensitive observation.

So was there a new pedagogy? Yes and no; it all depended on how you looked at the situation.

This is the situation we have now reached with research. If there is progress on this topic, it has been to recognise the complexity of the problem, rather than to provide a simple answer. In various ways, this complexity is played out across the articles included in this issue. Malcom Rutters describe work that seeks to preserve the ideals of education but realise them in new forms. A. Kamil Mahmood and Elaine Ferneley are, arguably, involved in trying to establish what the strategic purposes of particular educational traditions are, in order that new technologies can be designed to support them. Thiemo Müller-Kalthoff and Jens Möller describe a study that explores the match between a new set of learning operations and strategic aspirations to foster understanding. Rhona Sharpe, Greg Benfield and Richard Francis describe something slightly different, however - their exploration of the role of strategies in effecting organisational change initially seems to have little to do with the immediacy of pedagogy. Yet even here, the production of a document is being used to help groups of teachers work through what their educational strategy is, so that their subsequent use of technology supports rather learning, rather than just information management.

The challenge, then, is not to establish new pedagogies for e-learning in the simple sense of coming up with new things to do with learners. Instead, this more complicated picture requires a more conservative approach: finding out what teachers do and why, and then working out how technology can best be used to support that. This is not to deny the importance of developing innovative teaching techniques; there is certainly space in $A L T-\mathcal{f}$ for this, too. However, it is to call into question its relevance to the vast majority of teachers in higher education. If innovative research is all we do, we run the risk of not connecting, of producing nothing that is relevant and meaningful to their daily practices. It would be particularly ironic for our research to end up unread because we could not establish a connection. 